\title{
La confidentialité dans les courriers électroniques professionnels
}

\section{Philippe Millot}

\section{(2) OpenEdition}

1 Journals

Édition électronique

URL : http://journals.openedition.org/asp/244

DOI : $10.4000 /$ asp. 244

ISBN : 978-2-8218-0408-1

ISSN : 2108-6354

\section{Éditeur}

Groupe d'étude et de recherche en anglais de spécialité

Édition imprimée

Date de publication : 1 mars 2009

Pagination : 45-63

ISSN : 1246-8185

\section{Référence électronique}

Philippe Millot, « La confidentialité dans les courriers électroniques professionnels », ASp [En ligne], 55 | 2009, mis en ligne le 01 mars 2012, consulté le 02 novembre 2020. URL : http://

journals.openedition.org/asp/244 ; DOI : https://doi.org/10.4000/asp.244

Ce document a été généré automatiquement le 2 novembre 2020.

Tous droits réservés 


\title{
La confidentialité dans les courriers électroniques professionnels
}

\author{
Philippe Millot
}

\section{Introduction}

1 Depuis le milieu des années 1990, le développement des technologies de l'information conduit à une forte augmentation de la quantité et de la vitesse des informations divulguées au public: «Le temps qui sépare l'instant où l'information devient semipublique puis publique se restreint chaque jour davantage » (Kessler 2001: 34). Ainsi, les entreprises se rapprochent-elles du public en publiant sur Internet des informations autrefois considérées comme confidentielles ou réservées à un cercle restreint d'initiés telles que les chiffres d'affaires, les curriculum vitæ de leurs dirigeants ou le nom de leurs partenaires. Ce type d'information est cependant fortement contrôlé et sert autant d'outil d'information que d'outil de promotion. Il constitue en quelque sorte la façade officielle de l'entreprise et s'oppose en cela à sa communication privée ${ }^{1}$ dont une grande partie est composée d'échanges internes et externes tels que les conversations en face à face, les conversations téléphoniques ou encore la communication par courrier électronique. Ces échanges, parfois informels et peu contrôlés, sont des éléments constitutifs de l'intimité ${ }^{2}$ de l'entreprise. En ce qui concerne les courriers électroniques, cette intimité est cependant toute relative car la communication dans ce domaine fait l'objet de plus en plus de surveillance. En témoigne l'apparition de procès opposant certains employés peu prudents aux entreprises ${ }^{3}$. En résumé, si les entreprises publient désormais volontiers des données de plus en plus nombreuses sur leur fonctionnement, elles font en revanche preuve d'une grande vigilance vis-à-vis de leur communication privée.

2 Cette vigilance pose évidemment certains problèmes pour la recherche empirique, notamment en anglais des affaires. Les chercheurs qui étudient la communication privée en entreprise doivent gagner leur confiance, les rassurer et même lorsque la participation à une observation est acquise, ils constatent que les observés restent 
parfois réticents. Les propos de J. Holmes et M. Stubbe dans le cadre de leurs travaux sur les interactions en face à face au travail ${ }^{4}$ illustrent bien le problème :

Even though they knew that the content of their interactions would not be the focus of the research, our participants were initially very wary of losing control over any data that could potentially identify and compromise either individuals, or the organisations concerned. (Holmes \& Stubbe $2003: 20$ )

3 Cette réticence est d'autant plus problématique que les études sur corpus se multiplient, qu'elles nécessitent parfois de grandes quantités de données. Ces données étant difficiles à obtenir, les travaux portant sur les courriers électroniques en entreprise restent à ce jour peu nombreux (Mulholland 1999, Nickerson 1999, Kankaanranta 2005, Louhiala-Salminen 2002 et 2005, Gimenez 2006). Par conséquent, le domaine reste relativement méconnu des chercheurs en anglais des affaires malgré la place et le rôle tout à fait centraux que les courriels occupent désormais dans le quotidien de nombreux employés. Cette centralité est notamment illustrée dans l'étude de cas menée par L. Louhiala-Salminen décrivant la journée type d'un manager de multinationale :

The role of email in the structuring of the events during the day was crucial: the first two hours Timo spent working through the 95 messages that had arrived during the previous two days when he was out of office, and the majority of the subsequent interactions were also initiated by the 'box'. (Louhiala-Salminen 2002 : 217)

\section{Proposition de définition des « courriers électroniques professionnels »}

En France, la loi pour la confiance dans l'économie numérique ( $n^{\circ}$ 2004-575 datée du 21 juin 2004) définit le courrier électronique de la manière suivante :

On entend par courrier électronique tout message, sous forme de texte, de voix, de son ou d'image, envoyé par un réseau public de communication, stocké sur un serveur du réseau ou dans l'équipement terminal du destinataire, jusqu'à ce que ce dernier le récupère. (article IV)

5 La définition, purement technologique, ne dit rien quant au caractère privé ou public des messages. Pourtant, les litiges se multiplient, comme l'illustre ce texte issu de la proposition de loi visant à définir le courrier électronique professionnel ${ }^{5}$ :

Un cas symptomatique s'est ainsi présenté récemment, dans lequel la remontée classique d'informations par voie hiérarchique a fait apparaître de manière aiguë le risque de transgression de la confidentialité de ces éléments. Dans un premier temps, le responsable du service informatique d'une collectivité locale a adressé, par courriel, à tous les chefs de service, une note administrative leur demandant leurs besoins en matière de matériel et de logiciels, afin de préparer leur budget annuel. L'un d'entre eux a glissé, dans son message de réponse, intitulé «Re: budget ", des propos "insultants et délirants " à l'encontre du directeur général des services (DGS). Celui-ci l'apprenant par une tierce personne, a demandé au responsable de l'informatique de lui communiquer ce message, qu'il a versé au dossier administratif du fonctionnaire. En conséquence, le DGS a fait l'objet d'une mise en examen pour ces faits, sur la base du premier alinéa de l'article 432-9 du code pénal aux termes duquel le fait, par une personne dépositaire de l'autorité publique ou chargée d'une mission de service public, agissant dans l'exercice ou à l'occasion de l'exercice de ses fonctions ou de sa mission, d'ordonner, de commettre ou de faciliter, hors les cas prévus par la loi, le détournement, la suppression ou l'ouverture de correspondances ou la révélation du contenu de ces 
correspondances, est puni de trois ans d'emprisonnement et de 45000 euros

d'amende. (Proposition de loi du Sénat 385 2006)

6 D'après l'auteur de la proposition, le DGS soutient qu'il ne s'agissait pas un courrier privé mais d'un courrier professionnel, par conséquent "non soumis au secret ». Le problème, remarque l'auteur, est le flou juridique entourant la notion de courrier électronique professionnel. Dans le cadre de son projet de loi, l'auteur propose de définir cette notion de la manière suivante :

Est considéré comme courrier électronique professionnel, tout courrier électronique dont le titre ou le nom du répertoire dans lequel il est archivé, est relatif à l'organisation, au fonctionnement ou aux activités de l'entreprise, l'administration ou l'organisme qui emploie l'expéditeur ou le destinataire du dit courrier. Le courrier électronique professionnel n'est pas soumis au secret de la correspondance privée. (Proposition de loi du Sénat 385 2006)

Cependant, le fait que le titre et le lieu d'archivage soient pris comme caractéristiques des courriers électroniques est problématique. Notre corpus montre en effet que certains échanges, certes rares, ne possèdent pas de titre et sont pourtant des courriers professionnels. Le lieu d'archivage ne peut pas caractériser l'aspect professionnel d'un message dans la mesure où certains messages personnels pourraient y être stockés. Inversement, certains messages professionnels peuvent être envoyés sur des lieux d'archivages privés (dans le cas des travailleurs à domicile par exemple). En résumé, la proposition du Sénat semble imparfaite car elle caractérise le courrier professionnel par le contenant en partant du principe que l'enveloppe d'un message présuppose son contenu. Elle semble ainsi ignorer des aspects essentiels dans le domaine des interactions au travail : la nature et la fonction du contenu du message, le contexte situationnel dans lequel il a été envoyé et bien sûr ce que l'on peut entendre par «professionnel».

8 L'objectif ici est de proposer un premier cadrage sur ce qu'il est possible d'entendre par " courrier électronique professionnel ». Nous dirons tout d'abord qu'un message est professionnel si dans son contenu ${ }^{6}$, il se rapporte à des actions professionnelles (la notion "d'action professionnelle », très large, ne sera pas développée ici).Nous dirons ensuite qu'un message est professionnel par son contexte siles participants à l'échange sont caractérisés par une relation professionnelle, autrement dit, s'ils forment un groupe de discussion engagé dans le processus de réalisation des objectifs d'une ou plusieurs entreprises partenaires (exemple: fabrication de produits et publications afférentes). Le mot « professionnel » prend alors ici le sens d'expert ou de spécialiste ${ }^{7}$. Cette définition tend ainsi à exclure les interactions asymétriques (expert/profane) appartenant plutôt au domaine des interactions institutionnelles telles qu'elles ont été décrites par la littérature anglophone (Drew \& Heritage 1992). Dans ce cadre, un échange de courriers électroniques entre un employé et un client ne relèverait donc pas du domaine professionnel mais plutôt du domaine institutionnel. En revanche, un échange de courriers électroniques entre deux entreprises de fonctions différentes mais toutes deux expertes relèverait quant à lui du domaine professionnel.

9 Une définition du « courrier électronique professionnel » pourrait alors être formulée ainsi: "est considéré comme courrier électronique professionnel tout courrier électronique échangé dans le cadre d'actions professionnelles lorsque le groupe de discussion est défini par des relations professionnelles admises comme telle par ses membres ». Cette définition met en évidence deux groupes de personnes. Le premier 
groupe est constitué de membres d'une communauté de professionnels officiellement engagés dans la discussion. Comme il sera mentionné par la suite, cette discussion est confidentielle de facto. Le second groupe est constitué par toutes les personnes extérieures au premier groupe. Ce groupe inclut notamment les destinataires placés dans la zone " copie cachée " (BCC) $)^{8}$. Le groupe de discussion constituant un cadre de confidentialité, toute intrusion par une personne extérieure constitue une atteinte à cette confidentialité. Cette définition exclut bien entendu le cas des employés utilisant le réseau de l'entreprise pour communiquer à des fins personnelles. Enfin, cette définition permet de distinguer les courriers électroniques appartenant au réseau de l'entreprise (notion vaste incluant tous types de situations y compris la communication à des fins personnelles) des courriers électroniques professionnels (fournissant un cadre pragmatique observable au chercheur en anglais de spécialité).

\section{Les données}

\subsection{Nature des données}

10 Les données ont été recueillies sur une période de six mois, de juin à décembre 2007. Elles constituent un ensemble de 149 messages répartis dans vingt échanges de courriers électroniques en langue anglaise provenant de dix entreprises différentes toutes situées en France mais d'origines nationales diverses (britannique, américaine, française, allemande, suisse). Plusieurs domaines d'activité sont concernés : industrie automobile, industrie du meuble, hautes technologies (informatique et électronique), ressources humaines et finance. Les participants aux échanges sont des cadres dirigeants, des cadres exécutants et des chefs de projet. Si les participants à l'étude sont tous francophones, les échanges impliquent également des employés de langue maternelle différente. Les données regroupent des échanges dyadiques et en groupe, endogènes et exogènes.

\subsection{Méthode de recueil}

11 Les données ont été recueillies grâce à des contacts personnels. Les participants sont d'abord dirigés vers un blog édité par le chercheur. Ce blog présente la démarche de recherche, une charte de confidentialité et propose une méthode de recueil des données. Les employés acceptant la charte ont l'assurance que toutes les données permettant la reconnaissance des employés et des entreprises seront anonymées. Les participants transmettent ensuite par voie électronique des échanges en fonction de deux critères principaux: les échanges doivent être en anglais et représentatifs des habitudes professionnelles des employés. Afin de réduire les problèmes de confidentialité au minimum, les participants sont encouragés à puiser des échanges dans leurs archives dites "périmées", c'est-à-dire des échanges issus d'anciens dossiers. En puisant dans leurs archives, les participants restent libres de leur choix ${ }^{10}$. Cependant, et malgré les précautions prises, un questionnaire de fin d'observation révèle que dans la plupart des cas, certains messages ont été écartés sous prétexte qu'ils étaient « trop confidentiels ».

12 La méthode utilisée révèle une attitude ambivalente des employés envers leurs propres échanges. À quelques exceptions près, les employés acceptant l'observation sont tout 
d'abord enthousiastes à l'idée de participer à la recherche, puis, très rapidement, ils font preuve de réticences à l'idée de transmettre des données. Nous citerons l'exemple d'une participante qui, à la fin du recueil de données, nous confie malgré toutes les précautions prises en amont : «je compte sur votre discrétion ». Nous interpréterons ce passage de l'enthousiasme à la réticence comme un moment critique où le participant prend conscience que la transmission des données est contraire aux chartes de confidentialité ayant été signées à l'embauche ou lors de la signature de contrats.

\subsection{Discussion}

Les problèmes de confidentialité rencontrés ici sont tout à fait similaires à ceux posés dans d'autres enquêtes dans le domaine, que celles-ci portent sur les interactions en face à face ou sur les productions écrites des employés. Sur ce point, certains chercheurs adoptent une position radicale. Nous citerons par exemple J. C. Gimenez qui demande à tous les participants à un échange, qu'ils soient initiateurs, destinataires directs ou indirects, l'autorisation d'être observés : «In this particular instance, it seemed the right thing to do to ask for the consent of all the people whose names appeared in the embedded emails " (2006: 159). Cette position est certes éthiquement tout à fait légitime et applicable dans une enquête restreinte, mais nous pensons qu'elle constitue une entrave majeure à la conduite de travaux de plus grande ampleur ${ }^{11}$. L'enquête de terrain doit en effet trouver un équilibre tenable entre la nécessaire intrusion du chercheur dans le monde de l'entreprise et l'éthique liée à toute recherche dans ce domaine. En établissant une charte de confidentialité, en anonymant les données, en facilitant l'accès à l'information sur la recherche, et en laissant aux participants la possibilité de choisir les données à transmettre, nous pensons créer un cadre éthique et favorable à la conduite de l'enquête. Ces mesures ont par ailleurs l'avantage de rassurer les auteurs, qui, pour prendre leur point de vue, transmettent des données à distance à un destinataire-chercheur dont ils ne comprennent pas toujours très bien les motivations. Ces mesures ont également l'avantage d'impliquer les participants dans la recherche. Nous pensons en effet que l'implication active, comme le fait de laisser aux auteurs la liberté de choisir les données à transmettre, contribue à la qualité du corpus. Cette liberté mène tout d'abord les auteurs à réfléchir sur leur propre expérience. Elle génère ensuite des discussions avec le chercheur dont le résultat est bien souvent une atténuation de la peur de transmettre des données sensibles.

En résumé, nous appliquons le principe de D. Cameron et al. : " research on, for, and with our participants» (Cameron et al. 1992: 22). Ce principe nous rappelle toutefois que la constitution d'un corpus de données issues du monde professionnel reste limitée par son origine pratique: "There is, after all, no absolute freedom, independence or objectivity which privileges one form of research over another. Real life always comes rampaging in." (Sarangi \& Roberts 1999 : 40). Cette « vie réelle » sera ainsi prise comme point de départ pour l'élaboration de notre cadre d'analyse. 


\section{Cadres juridique et théorique}

\subsection{Cadre juridique}

15 La confidentialité est définie ainsi : «maintien du secret concernant des informations (dans une administration, dans un système informatisé...), dans le dessein d'empêcher une utilisation frauduleuse » (Rey2005: 1765). D'après cette définition, transmettre des données à une personne extérieure à l'entreprise consisterait à trahir le secret, c'est-àdire à révéler ce que l'entreprise ne souhaite pas dévoiler au public. Les entreprises font d'ailleurs régulièrement figurer ce type d'avertissement dans leurs échanges :

Exemple 1. Message d'avertissement rencontré dans le corpus

This e-mail may contain confidential information and/or copyright material. This e-mail is intended for the use of the adressee only. Any unauthorized use may be unlawful. If you receive this e-mail by mistake, please advise the sender immediately by using the reply facility in your e-mail software.

Si ce type de message ne possède pas de valeur légale proprement dite, il résulte généralement d'un accord de confidentialité passé en amont entre les entreprises partenaires. En premier lieu, cet accord précise l'objet de la confidentialité, comme le montre l'exemple suivant :

Exemple 2. Extrait d'un accord de confidentialité

Confidential information shall include all data, materials, products, technology, computer programs, specifications, manuals, business plans, software, marketing plans, financial information, and other information disclosed or submitted, orally, in writing, or by any other media, to Recipient by Owner.

17 En second lieu, il délimite la zone de confidentialité, très large dans certains cas, située entre un " propriétaire d'information» (« Owner ») et un " destinataire» («Recipient »). Le destinataire, comme le propriétaire, peut être une personne physique ou une personne morale comprenant toutes les entités que cette dernière est susceptible de représenter :

Exemple 3. Extrait d'un accord de confidentialité

For purposes of this Agreement, the term 'Recipient' shall include Recipient, the company he or she represents, and all affiliates, subsidiaries, and related companies of Recipient. [...] For purposes of this Agreement, the term 'Representative' shall include Recipient's directors, officers, employees, agents and financial, legal, and other advisors.

Dans le cas des courriers électroniques, certaines entreprises précisent que les destinataires sont à la fois les personnes placées dans la zone « $\mathrm{A} / T o$ » et celles placées dans la zone « $\mathrm{CC}$ » :

Exemple 4. Message d'avertissement sur un forum de discussions entre professionnels ${ }^{12}$

The information contained in this e-mail and any accompanying documents is confidential, may be privileged, and is intended solely for the person and/ or entity to whom it is addressed (i.e. those identified in the 'To' and 'cc' box). 

lequel se trouvent le propriétaire et le destinataire. L'extrait suivant montre que ce contexte est caractérisé par une relation professionnelle et/ou une transaction entre les deux parties :

Exemple 5. Extrait d'un accord de confidentialité

Recipient may disclose Owner's Confidential Information to Recipient's responsible Representatives [...] only to the extent necessary to evaluate or carry out a proposed transaction or relationship with Owner.

Par conséquent, les messages transmis par les participants sont tous confidentiels de facto. Ils s'inscrivent en effet dans une zone à l'écart du public que nous pourrions définir ici comme l'ensemble des personnes non engagées dans une relation professionnelle ou une transaction avec le propriétaire de l'information confidentielle.

\subsection{Cadre théorique}

E. Goffman (1959), sociologue américain, propose de classer les secrets en trois catégories. La première est celle des secrets « inavouables ", c'est-à-dire les faits critiques que l'on cache. La deuxième catégorie est celle des secrets "stratégiques »: " these pertain to intentions and capacities of a team which it conceals from its audience in order to prevent them from adapting effectively to the state of affairs the team is planning to bring about" (Goffman 1959: 142). Enfin, la troisième catégorie est celle des secrets "d'initiés ": " these are the ones whose possession marks an individual as being a member of a group and helps the group feel separated and different from those individuals who are not 'in the know'» (Goffman 1959: 142). Parce que les échanges électroniques professionnels véhiculent des données secrètes, nous formulerons l'hypothèse que les trois types de secret proposés par Goffman peuvent être adaptés à notre corpus.

Les secrets inavouables sont peu nombreux et correspondent généralement à de courtes digressions dans le cadre d'échanges en interne.

Exemple 6. Confidence suite au dysfonctionnement d'un produit vendu au Japon

\{name 1\}, \{name 2\},Here is the first feedback from one customer we tried that your HT blade: In customer's words: 'they sucked' and were removed after only running one week.

On trouve également ce type de secret dans les échanges de type client-fournisseurs. Il s'agit alors d'échanges réparateurs suite à des problèmes de commande.

Les secrets stratégiques se trouvent dans la plupart des échanges internes ou avec les entreprises partenaires. Il s'agit des échanges dont l'objet est de mettre en place des projets (produits ou présentations à la clientèle, par exemple). Ils correspondent à un ensemble de données telles que les prix, les références et les noms de partenaires. Contrairement aux secrets inavouables, il n'existe pas de passage particulièrement sensible dont la révélation pourrait porter préjudice à l'employé ou à l'entreprise. Les secrets stratégiques sont en fait dilués dans un ensemble de messages et pièces jointes dont la divulgation pourrait avantager la concurrence.

Les secrets d'initiés forment une catégorie globale comprenant l'ensemble des échanges. En effet, tous les employés impliqués dans les échanges (en tant que 
participants actifs ou simples "témoins ») font partie d'un groupe d'initiés. Ceux-ci partagent une langue ainsi qu'un style d'expression propres à l'entreprise.

Exemple 7. Demande d'éclaircissement entre un ingénieur américain et un ingénieur français issus de la même entreprise de haute technologie

"Sacrifice of the offblock bags of the module saturating the EBS"? How is this something to show ADT? It sounds like normal functionality to me.

I thing it should be possible to test the behavior with the « Mode Degrade » test to verify it is working correctly.

quiconque ne partage pas l'univers de références situé entre l'émetteur et le destinataire. L'univers est ici caractérisé par un lexique spécialisé (« offblock bags »), des sigles et des marqueurs de fléchages (" the module», " how is this ») renvoyant à la fois à la situation, au contexte du discours et à la connaissance experte d'un domaine. Les phrases sont par ailleurs juxtaposées sans lien apparent laissant à penser qu'en plus de cet univers de référence, il existe un style d'expression très direct accepté par les deux participants ${ }^{13}$. La réponse est d'ailleurs la suivante :

Exemple 8

Outre l'univers de référence et la langue, nous constatons que ces groupes discutent sur d'autres médias recourant ainsi à des genres comme la conversation téléphonique ou la réunion professionnelle.

Exemple 9. Extrait d'un échange entre ingénieurs

Hi \{name 1\}, 2 weeks ago during a meeting w/ AsP, we (name 2 \& I) were questioned about RH\#675 (actually, I've seen that its criticity is 7, which is strange, anyway) and all RH about « sacrifice » in general (RH\#64321 ...).

« 2 weeks ago during a meeting" dénote que le courrier électronique fait suite à une réunion de travail. Parallèlement, le commentaire entre parenthèses («actually [...] which is strange anyway») rappelle fortement le style que pourrait adopter le même participant en réunion.

9 D'une part, cet exemple confirme les conclusions de J. Mulholland (1999) qui définit le courrier électronique comme complémentaire d'autres genres ${ }^{14}$. D'autre part, il illustre la perception holistique de la communication professionnelle dans l'étude de cas proposée par Louhiala-Salminen :

Throughout the day spoken and written communication were totally intertwined, there was hardly any activity in either mode where the other would not be present as well; many of the phone calls were to confirm an issue in an e-mail message, email messages refered to phone calls, and they were constantly discussed in face-toface communication with colleagues. This finding is interesting. Bearing in mind the present views of literacy discussed above we could say that in this type of business discourse it is the ongoing and recurring interplay where the business professional utilizes his literacies both in speech and writing and in the media used - including intermingled usage - to achieve his purposes. (2002:217)

Les courriels formeraient donc une zone d'intersection entre différents canaux où les groupes de discussion tendent vers des but communicatifs « complexes, multiples et évasifs" (Swales 2004). Cette complexité et cette multiplicité sont illustrées par l'exemple précédent où trois buts sont visés simultanément: 
31 - l'échange d'informations relatives aux activités professionnelles (ici la résolution d'un problème technique), rappelant «l'orientation informationnelle » des messages (Kankaraanta 2005) ;

32 - l'entretien des relations professionnelles (l'informalité créant une certaine solidarité). rappelant « l'orientation relationnelle » des messages (Kankaraanta 2005);

33 - le partage de secrets d'initiés (vocabulaire spécialisé, utilisation d'abréviations particulières tels que « $w /$ "pour « with», références employées comme des noms). Cet objectif rappelle fortement le propos de Swales (1990) sur les communautés de discours : "[discourse communities] tend to separate people into occupational or specialityinterest groups » $(1990: 24)$.

34 En résumé, les échanges issus de notre corpus permettent à des groupes spécialisés d'échanger des informations secrètes aux objectifs multiples et dans un style partagé. Ceci suggère que ce medium pourrait servir de support à des "genres occlus » dont Swales (1996) donne une définition dans le contexte universitaire : « academic occluded genres are, in part, those which support the research publication process but are not themselves part of the research record " (Swales 1996 : 45). Si nous reformulons cette définition dans le cadre de l'anglais des affaires, nous obtenons la proposition suivante: "business occluded genres are, in part, those which support the business publication process but are not themselves part of the business record ". Par "support the business publication process ", nous entendons que les textes "occlus" ou officieux ont pour fonction de contribuer au processus de publication des données ${ }^{15}$. Le terme "support» est par ailleurs suffisamment large pour englober les deux parties du processus de publication: les textes préparatoires d'une part et les textes faisant suite à la publication des données officielles d'autre part (plaintes, ajustements, etc.). La figure 1 illustre la place que tiennent les courriers électroniques dans ce processus.

Figure 1. Place des textes confidentiels dans le processus de publication

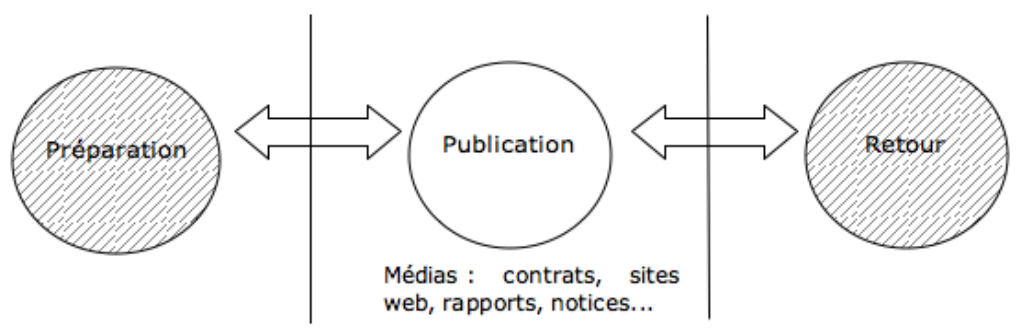

Dans le corpus étudié, la façon dont les courriers électroniques se répartissent figure au tableau 1. 
Tableau 1. Répartition des échanges du corpus en fonction de leur place dans le processus de publication

\begin{tabular}{|c|c|c|}
\hline \multicolumn{3}{|c|}{ Préparation des textes publiés } \\
\hline Domaine & $\begin{array}{c}\text { Objets des échanges (Textes cachés } \\
\text { /occlus) }\end{array}$ & $\begin{array}{c}\text { Textes } \\
\text { officiels }\end{array}$ \\
\hline Informatique & $\begin{array}{l}\text { organisation d'une démonstration de logiciel } \\
\text { à un client }\end{array}$ & $\begin{array}{l}\text { présentation de } \\
\text { produit }\end{array}$ \\
\hline RH & $\begin{array}{l}\text { organisation du paiement d'un salarié } \\
\text { français au Danemark }\end{array}$ & $\begin{array}{l}\text { bulletin de } \\
\text { salaire danois }\end{array}$ \\
\hline RH & $\begin{array}{l}\text { demande d'informations pour publier un } \\
\text { poste en Belgique }\end{array}$ & $\begin{array}{l}\text { publication du } \\
\text { poste }\end{array}$ \\
\hline Automobile & commande de matériel & facture \\
\hline Finance & annonce d'accord entre deux entreprises & contrat \\
\hline Finance & $\begin{array}{l}\text { demande d'information sur possibilités de } \\
\text { crédit }\end{array}$ & contrat \\
\hline Industrie & bilan de tests techniques & $\begin{array}{l}\text { notice } \\
\text { technique }\end{array}$ \\
\hline Ameublement & calcul du prix d'un futur produit & catalogue \\
\hline
\end{tabular}

Retour sur les textes publiés

\begin{tabular}{|l|l|l|}
\hline \multicolumn{1}{|c|}{ Domaine } & \multicolumn{1}{|c|}{$\begin{array}{c}\text { Objets des échanges (Textes } \\
\text { cachés/occlus) }\end{array}$} & \multicolumn{1}{c|}{$\begin{array}{c}\text { Textes } \\
\text { officiels }\end{array}$} \\
\hline Industrie & réparation suite à un défaut de matériel & $\begin{array}{l}\text { conditions de } \\
\text { vente }\end{array}$ \\
\hline Industrie & erreur de commande & $\begin{array}{l}\text { contrat de } \\
\text { vente }\end{array}$ \\
\hline Industrie & annonce de livraison & $\begin{array}{l}\text { contrat de } \\
\text { vente }\end{array}$ \\
\hline
\end{tabular}

\section{Résultats}

\subsection{La nature de la confidentialité dans les échanges}

Comme il a été déjà mentionné, les courriels professionnels possèdent tout d'abord une orientation informationnelle et véhiculent à ce titre des informations considérées comme « sensibles » par les participants : le nom des entreprises partenaires ( $21 \%$ des messages), les références à des dossiers ou des produits (47\%) et des noms de personnes (5\%). À cela, il faut ajouter que le medium lui-même véhicule des données confidentielles: les adresses électroniques professionnelles (généralement sous la forme prénom.nom@entreprise.com) permettent en effet dans la plupart des cas l'identification des émetteurs et des destinataires. Les courriels possèdent enfin une orientation relationnelle mise en œuvre par différents degrés de formalité et d'informalité.

\subsection{Formalité, nature et enjeux}

37 À quelques exceptions près, les textes issus de notre corpus sont caractérisés par une certaine homogénéité stylistique. Ainsi, $90 \%$ des messages correspondent aux commentaires de J. Gains (1999) sur le style des courriels commerciaux : «In general, the stylistic register employed by the writers of the commercial e-mail examples appeared to be largely consistent and may be characterised as using the semi-formal tone of co-operative business colleagues » (Gains 1999 : 86).

Dans la lignée de ses travaux, les nôtres tendent à montrer que les échanges hybrides constituent la règle et que les messages purement formels ou informels sont 
l'exception. L'analyse stylistique détaillée des messages ne constituant pas l'objet de recherche ici, nous dresserons le tableau des caractéristiques les plus pertinentes pour la question de la confidentialité.

Contrairement aux idées reçues sur les courriers électroniques, le recours à la formalité est fréquent et remplit des objectifs précis dans la mise en scène des rapports professionnels ${ }^{16}$. Elle se réalise par un degré de structuration plus ou moins élevé, mais observé dans la très grande majorité des messages de notre corpus. Ainsi, à l'instar des lettres commerciales, la plupart des messages sont composés d'une section d'ouverture suivie d'une section propositionnelle et s'achèvent généralement par une section de fermeture. Les sections d'ouverture et de fermeture peuvent prendre des formes diverses, selon le degré de connaissance des interlocuteurs ( « Hi », « Hello », « To all », « Dear » + identification, «Best regards », ou encore «Thks! »). La section propositionnelle offre de grandes variations stylistiques que nous ne détaillerons pas ici. Nous signalerons à ce stade la présence de style formel pour faire des requêtes («could you ...", " please ...»), faire des remerciements, et de manière plus générale, accomplir des actes risquant de porter atteinte à l'image positive que les acteurs sociaux en représentation souhaitent donner.

Exemple 10. Extrait d'un courriel envoyé par un manager français à son client japonais suite à un important problème de commande

Dear Mr. \{name\}, Thank you for your fast answer, it will be a great pleasure to meet as soonas possible with \{company name\} to discuss about the qualityissues they have met with our material. We believe the discussions with the customers is the right way to progress in order to meet and exceed the customer expectations.

La formalité du message est d'abord concrétisée par la formule de politesse « Dear Mr+ nom » suivie d'un remerciement de façade. Une phrase telle que «We believe ... the customer expectations " rappelle fortement le style promotionnel des entreprises en représentation sur la toile ${ }^{17}$. Dans ce cas, nous dirons que la formalité remplit deux objectifs. Elle place en premier lieu les acteurs sociaux sur la scène des relations clients-fournisseurs, "Dear $M r+$ nom » fournissant le premier élément du décor. Elle permet en second lieu au manager de jouer le rôle attendu dans ce genre de situation. En effet, en rappelant la responsabilité morale de l'entreprise ( we believe ... the right way to progress »), l'auteur du message présente implicitement des excuses tout en suggérant un retour au déroulement normal de la transaction.

La formalité, ici mise en œuvre dans un message envoyé dans les coulisses, comporte une valeur symbolique car elle permet la mise en scène de la politesse dans le but de réguler les rapports parfois conflictuels au travail. Nous pourrions dire qu'elle contribue en quelque sorte à l'intimité de l'entreprise, ce qui expliquerait, du moins en partie, les réticences des employés à transmettre les données même anonymées.

\subsection{L'informalité, nature et enjeux}

L'informalité se concrétise tout d'abord par la présence de certains outils dialogiques tels que les questions directes et la présence de certaines expressions à fonction essentiellement phatique. Les exemples suivants sont un condensé de ces caractéristiques tout à fait régulières dans le corpus.

Exemple 11 
ok, \{name\}. Could you please confirm the dimensions of your offer: thickness 4.125" x dia 9.05" ? Price: \$26,220.00 piece. ?Thank you. Best regards, \{name\}

Exemple 12

Fair enough. I am not over surprised as it is exactly the sort of problems we had in 2005.

L'informalité se concrétise également par le style familier, les abréviations et les ellipses, comme le montre l'exemple 13.

Exemple 13

One thing I wantto add is we need to discuss in a detailed way with the technical guy from \{company name\} the technical scope of this demo.

dernière caractéristique fréquemment rencontrée est un certain degré d'oralisation de l'écrit rappelant fortement le style des conversations privées. L'exemple 14 montre deux phénomènes courants dans le corpus. Le premier est l'utilisation de "the» comme outil de repérage situationnel. L'énonciateur et le coénonciateur, parce qu'ils sont situés dans le cadre d'une correspondance privée, ont une connaissance partagée de l'objet fléché (ici la pièce jointe) qu'il n'est pas nécessaire de rappeler explicitement. Le second phénomène est un éloignement des normes grammaticales à l'écrit, notamment en matière de ponctuation.

Exemple 14

Please see the attachment, is that what you need?

Ces exemples tendent à prouver que le style informel est relativement proche de celui des conversations ordinaires au travail décrites par la recherche (Gumperz 1982, Drew \& Heritage 1992, Holmes \& Stubbe 2003). Ils sont la trace d'un discours peu planifié laissant apparaître les hésitations, les doutes et les questions des employés dans la réalisation de leurs actions professionnelles. Ces exemples constituent alors probablement une menace pour la face officielle des entreprises soucieuses de l'image de fiabilité qu'elles souhaitent donner au public.

Les échanges sont caractérisés par une certaine intimité faite de doutes, de réparations et d'autres secrets partagés. Ils possèdent à ce titre un potentieldestructeur (Goffman 1959) dans la mesure où la révélation des secrets pourrait porter atteinte à l'image de confiance et d'efficacité que souhaitent donner les entreprises en représentation. Ce potentiel destructeur, fondé sur une confidentialité de façade, liée à l'orientation informationnelle des messages, et une confidentialité plus profonde liée à l'orientation relationnelle, expliquerait d'ailleurs les réticences des employés à transmettre leurs échanges au chercheur, même lorsque ce dernier promet de garantir l'anonymat. En effet, le nettoyage des données «sensibles» n'est qu'une opération superficielle révélant les données profondément problématiques que sont les comportements secrets dans les coulisses. Cependant, dans la lignée des travaux précédents (Mulholland 1999, Gimenez 2000, Crystal 2001), notre étude montre que l'intimité entre acteurs est toute relative car la crainte de perdre la face, ajoutée à la peur qu'un message puisse "s'échapper" pour être transmis urbi et orbi à des destinataires inconnus, forcerait les acteurs à faire preuve d'auto-contrôle. Si les échanges ont lieu dans les coulisses, la scène, elle, n'est jamais loin. 


\section{Conclusion}

\section{électroniques, pose cependant le problème des frontières entre les différents domaines} de la langue anglaise, voire entre les langues elles-mêmes. La première zone problématique est la frontière entre la langue spécialisée et la langue générale. La seconde zone frontalière est celle de l'anglais comme lingua franca (Jenkins 2004), c'està-dire de l'anglais comme langue de travail entre les natifs et les non-natifs d'une part, et entre les non-natifs entre eux d'autre part.

51 Le corpus, aussi réduit soit-il, montre en effet que les échanges purement techniques n'existent pas dans la mesure où les locuteurs doivent par exemple "prendre la parole » et « la rendre » en recourant à un lexique appartenant à la langue « de tous les jours ». Cette langue ordinaire donnant un aspect anodin aux échanges permet aux employés de faire des affaires. Elle appartient à ce titre à l'anglais "des affaires » caractérisé par Pickett comme "a lot nearer the everyday language spoken by the general public than many other segments of ESP» (Pickett 1986 dans St John 1996: 6). Parallèlement, les échanges purement généraux n'existent pas dans la mesure où le point de départ d'une discussion est toujours lié aux dossiers spécialisés de l'entreprise faisant ainsi des courriers électroniques un terrain fertile pour la langue spécialisée. Les échanges entre ingénieurs, par exemple, prouvent que ces derniers partagent non seulement des lexiques spécialisés mais également certaines façons de parler renforçant ainsi le sentiment d'appartenance à une communauté tout en créant un sentiment d'exclusion chez le lecteur profane. Ceci suggère que le discours spécialisé permet certes l'échange de données sensibles mais fournit également un outil de contrôle aux frontières entre les communautés spécialisées et celle des profanes. Cette 
propriété semble centrale aux échanges par courriers électroniques, rappelant le discours "sentinelle» évoqué par Holmes (2007: 1993): "Gatekeeping involves monitoring boundaries and is typically accomplished through discourse, especially in organizational contexts ». Une première étude en anglais de spécialité consisterait donc à rendre compte de la nature et du rôle des discours spécialisés dans les courriers électroniques. Parallèlement, et parce qu'elle est indissociable de la première catégorie, une seconde étude consisterait à explorer la nature et la fonction de cette langue « ordinaire » traditionnellement délaissée au profit de la première. L'objectif ici est de pallier la lacune relevée par Sarangi et Roberts: «And it is this everydayness that takes place backstage which often tends to be ignored by workplace researchers " (Sarangi \& Roberts 1999 : 22).

Le corpus révèle également que les courriers électroniques se situent dans une zone frontalière entre un anglais "officiel ", "standard", et une langue pratiquée par les employés, faite d'erreurs, d'approximations et d'emprunts à d'autres langues que l'anglais. Ce phénomène de mélange des variétés de l'anglais représenterait une caractéristique de cette langue "dans les coulisses" où la parole est contrôlée de manière relative. Il serait influencé par une constellation de facteurs tels que le statut de l'anglais comme lingua franca, l'urgence du dossier, la culture de l'entreprise, et de manière plus générale, le medium lui-même. Une troisième étude consisterait alors à caractériser cette langue située au carrefour de l'anglais standard, l'anglais comme lingua franca et l'anglais « des nouveaux réseaux de communication » (Crystal 2001).

\section{BIBLIOGRAPHIE}

Angouri, J. et N. Harwood. 2008. « This is too formal for us ...: A case study of variation in the written products of a multinational consortium ». Journal of Business and Technical Communication 22, 38-64.

Cameron D., E. Frazer, P. Harvey, M. B. H. Rampton et K. Richardson. 1992. Researching Language. Issues of Power and Method. Londres : Routledge.

Crystal, D. 2001. Language and the Internet. Cambridge : Cambridge University Press.

Drew, P. et J. Heritage (dir.). 1992. Talk at Work. Interaction in Institutional Settings. Cambridge : Cambridge University Press.

Gains, J. 1999. «Electronic mail - A new style of communication or just a new medium? » English for Specific Purposes 18/1, 81-101.

Gimenez, J. C. 2000. «Business e-mail communication: Some emerging tendencies in register. » English for Specific Purposes 19/3, 237-251.

Gimenez, J. 2006. « Embedded business emails: Meeting new demands in international business communication ». English for Specific Purposes 25/ 2, 154-172.

Goffman, E. 1959. The Presentation of Self in Everyday Life. New York : Anchor Books.

Gumperz, J. J. 1982. Discourse Strategies. Cambridge : Cambridge University Press. 
Holmes, J. 2007. « Monitoring organisational boundaries: Diverse discourse strategies used in gatekeeping. » Journal of Pragmatics 39, 1993-2016.

Holmes, J. et M. Stubbe. 2003. Power and Politeness in the Workplace. Harlow : Pearson Education Limited.

Jenkins, J. 2004. «EFL at the gate. The position of English as a lingua franca ». The European Messenger 13/2, 63-69.

Kankaanranta, A. 2005. « Hej seppo, could you please comment on this? " Internal Email Communication in Lingua Franca English in a Multinational Company. Jyväskylä, Finlande : Jyväskylä University Press.

Kessler, D. 2001. « L'entreprise entre transparence et secret ». Pouvoirs 97, 33-46.

Loudermilk, B. C. 2007. « Occluded Academic Genres: An Analysis of the MBA Thought Essay ». Journal of English for Academic Purposes 6, 190-205.

Louhiala-Salminen, L. 2002. " The fly's perspective: discourse in the daily routine of a business manager ». English for Specific Purposes 21/2, 211-231.

Louhiala-Salminen, L. et A. Kankaanranta. 2005. « English as a lingua franca in nordic corporate mergers: Two case companies ». English for Specific Purposes 24/4, 401-421.

Markus, M. L. 1994. «Electronic mail as the medium of managerial choice ». Organization Science 5/4, 502-527.

Mulholland, J. 1999. « E-mail: Uses, issues and problems in an institutional setting ». In F. Bargiela-Chiappini et C. Nickerson (dir.) Writing Business: Genres, Media and Discourses. Harlow, GB : Longman, 57-84.

Nickerson, C. 1999. « The use of English in electronic mail in a multinational corporation ». In F. Bargiela-Chiappini et C. Nickerson (dir.) Writing Business: Genres, Media and Discourses. Harlow, GB : Longman, 35-56.

Petit, M. 2002. «Éditorial ». ASp 35-36,1-3.

Rey, A. 2005. Dictionnaire culturel en langue française. Paris : Dictionnaires Le Robert.

Sarangi, S. et C. Roberts. (dir.). 1999. Talk, Work and Institutional Order: Discourse in Medical, Mediation and Management Settings. New York : Mouton de Gruyter.

St John, M .J. 1996. « Business is booming: Business English in the 1990s ». English for Specific Purposes 15/1, 3-18.

Swales, J. M. 1990. Genre Analysis: English in Academic and Research Settings. Cambridge : Cambridge University Press.

Swales, J. M. 1996. " Occluded genres in the academy: The Case of the submission letter ». Pragmatics \& beyond 41, 45-58.

Swales, J. M. 2004. Research Genres: Explorations and Applications. Cambridge : Cambridge University Press.

Référence juridique

Proposition de loi visant à définir le courrier électronique professionnel. Sénat. 385. Session

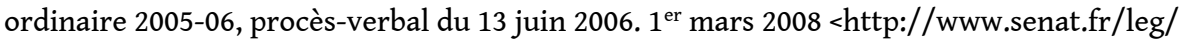
ppl05-385.htm>. 


\section{NOTES}

1. Par communication privée, nous entendons non seulement la communication interne à l'entreprise ou au groupe, mais également la communication vers les entreprises partenaires que sont les clients et les fournisseurs.

2. L'intimité et son adjectif "intime " trouveront un sens ici plutôt littéraire. Les écrits intimes d'une entreprise ou d'un groupe d'employés seront donc ceux qui touchent la vie privée de cette entreprise ou de ce groupe. À ce titre, ils ne sont pas destinés à être dévoilés au public.

3. C'est le cas de Dow Chemical aux États-Unis qui, en 2000, renvoie vingt-quatre employés suite à des échanges de courriers portant atteinte à l'entreprise <http://www.cnet.com/>.

4. Ces travaux font partie du Wellington Language Project, vaste programme de recherche en analyse du discours dans le domaine des organisations publiques et privées néo-zélandaises.

5. Texte proposé par François Marc, à l'issue de la session ordinaire de 2005-06, annexe au procès-verbal de la séance du 13 juin 2006 (Proposition de loi visant à définir le courrier électronique professionnel) <http://www.senat.fr/leg/ppl05-385.html>.

6. Par contenu, nous entendons l'objet et le corps du message.

7. Ce sens de «professionnel » se rapprocherait donc de «professional» dans la mesure où les « professions » en anglais sont les métiers du savoir.

8. Ces derniers possèdent en effet le simple statut d'observateur et ne peuvent pas être connus, donc admis, par le groupe de discussion.

9. <http://s123734617.onlinehome.fr/bienvenue/>.

10. Approche dite « hands off» (Holmes et Stubbe 2003) et reprise par Angouri et Harwood (2008) où le chercheur laisse aux participants l'entière liberté de choisir quelles données transmettre.

11. Ce corpus restreint fait partie d'un corpus plus large faisant actuellement l'objet d'une thèse.

12. <http://www.ittoolbox.com/>.

13. La juxtaposition des énoncés rappelle l'effet d'ajout (« add-on effect ») décrit par Mulholland (1999).

14. L'auteur considère en effet que le courrier électronique est un genre nouveau.

15. Dans notre domaine, il s'agit des données officielles transmises en interne ou en externe. Elles peuvent être privées (contrats de vente, contrats d'embauche, etc.) ou publiques (catalogues, offres d'embauche, etc.). Dans cette perspective, la présentation d'un produit à l'oral peut être considéré comme une forme de publication dans la mesure où elle porte le produit à la connaissance d'une communauté, voire de tous.

16. Nous faisons ici référence à la métaphore dramaturgique de Goffman (1959). L'auteur y distingue la parole ordinaire échangée dans les coulisses (backstage talk) de celle échangée par des personnes en représentation devant un public (frontstage talk).

17. «BT Continues to Exceed Customer Expectations » est par exemple le titre d'un article tiré du site BT Americas Horizon <http://www.btamericashorizon.com/BTAmericasHorizon/Article. asp?Article Code $=10079323 \&$ EditionCode $=25483528)>$.

\section{RÉSUMÉS}

Les échanges de courriels en entreprise relèvent du domaine privé et, à ce titre, sont tous confidentiels. À partir d'un corpus constitué en 2007, cet article propose une observation de la confidentialité dans les échanges en anglais issus de dix entreprises situées en France. Une 
nouvelle définition des courriels professionnels est proposée. La confidentialité est ensuite envisagée comme une caractéristique centrale dans les échanges. Cette confidentialité, issue du partage des secrets d'initiés, semble alors indiquer que les courriels professionnels véhiculent des genres « occlus » en anglais de spécialité.

As corporate email exchanges belong to the private domain, they are all confidential. Based on a corpus constructed in 2007, the article aims at observing confidentiality in the emails in English from ten companies located in France. First, a new definition of corporate emails is proposed. Confidentiality is then studied as a central feature in the exchanges. This confidentiality, being the result of "inside" secrets, suggests that corporate emails serve as a channel for "occluded" genres in English for Specific Purposes.

\section{INDEX}

Mots-clés : confidentialité, courriel professionnel, genre occlus, secret d'initié

Keywords : confidentiality, corporate email, inside secret, occluded genre

\section{AUTEUR}

\section{PHILIPPE MILLOT}

Philippe Millot enseigne l'anglais aux étudiants en sciences humaines à l'Université de FrancheComté, où il dirige un centre de ressources en langues. Il prépare une thèse de doctorat à l'Université Grenoble 3 sur les échanges de courriers électroniques en anglais comme langue de travail dans les entreprises. philippe.millot@univ-fcomte.fr 